\title{
TISSUE-PRESERVING APPROACH TO EXTRACTING DNA FROM PARAFFIN-EMBEDDED SPECIMENS USING TISSUE MICROARRAY TECHNOLOGY
}

\author{
Mark E. Prince, MD, ${ }^{1 *}$ Mathew L. Ubell, MD, ${ }^{1 *}$ Jason Castro, BSc, ${ }^{1}$ Haruko Ogawa, MD, ${ }^{1}$ \\ Tetsuya Ogawa, MD, ${ }^{1}$ Ajita Narayan, MD, ${ }^{1}$ Augusto Paulino, MD, ${ }^{2}$ Andrew Cole, BA, ${ }^{1}$ \\ Gregory T. Wolf, MD, ${ }^{1}$ Mark A. Rubin, MD, ${ }^{2}$ Robert H. Lyons, PhD, ${ }^{3}$ Carol R. Bradford, MD, ${ }^{1}$ \\ Thomas E. Carey, PhD $^{1}$
}

${ }^{1}$ Department of Otolaryngology-HNS, University of Michigan, 1500 E. Medical Center Drive, Ann Arbor, Michigan, 48109. E-mail: mepp@umich.edu

${ }^{2}$ Department of Pathology, University of Michigan, Ann Arbor, Michigan

${ }^{3}$ DNA Sequencing Core, University of Michigan Cancer Center, Ann Arbor, Michigan

Accepted 10 August 2006

Published online 24 January 2007 in Wiley InterScience (www.interscience.wiley.com). DOI: 10.1002/hed.20547

\begin{abstract}
Background. DNA extracted from tumor cells or normal cells contained in formalin-fixed, paraffin-embedded tissues is widely used in many laboratories. The 2 most common procedures to isolate cells for DNA extraction from paraffin-embedded tissues are scalpel microdissection and laser capture microdissection. A new tissue- and time-conserving method for rapid DNA isolation from small cores taken from paraffin-embedded tissue blocks is described in this report.

Methods. DNA was extracted from small tissue cores collected from paraffin-embedded tissue blocks at the time of tissue microarray construction. The quality and quantity of the DNA extracted was compared to DNA collected by scalpel microdissection. DNA collected from tissue cores was used in polymer-
\end{abstract}

Correspondence to: M. E. Prince, University of Michigan, 1500 E. Medical Center Drive, 1904 Taubman Center, Ann Arbor, MI 48109.

*These authors contributed equally to this work.

Contract grant sponsor: National Cancer Institute Specialized Program of Research Excellence (SPORE); contract grant numbers: P50 CA69568, P50 CA97248, R01 CA83087, R01 DE13346, RO1 DE13346; contract grant sponsor: NIH NIDCD; contract grant number: P30 DC5188; contract grant sponsor: $\mathrm{NIH}$; contract grant number: T32 DC05356; contract grant sponsor: University of Michigan Bioinformatics Program; contract grant number: 379206

(c) 2007 Wiley Periodicals, Inc ase chain reaction (PCR) and loss of heterozygosity $(\mathrm{LOH})$ analysis.

Results. The quality and quantity of DNA obtained using tissue cores was comparable to DNA obtained by traditional methods. The tissue core method of DNA extraction preserves the tissue blocks from which the cores are extracted for future use. Adequate quantities of DNA can be successfully extracted from small segments of tissue cores and used for PCR. DNA isolated by tissue microdissection and the tissue core method were comparable when used to assess allelic heterozygosity on chromosome arm 18q.

Conclusion. The tissue core method of DNA isolation is reliable, tissue conserving, and time effective. Tissue cores for DNA extraction can be harvested at the same time as tissue microarray construction. The technique has the advantage of preserving the original tissue blocks for additional study as only tiny cores are removed. (c)2007 Wiley Periodicals, Inc. Head Neck 29: 465-471, 2007

Keywords: DNA extraction; tissue microarray; squamous cell cancer

Paraffin-embedded tissue is an excellent source of DNA. The stability of DNA preserved in paraffin after formalin fixation allows it to be successfully 
extracted from tissue stored for many years. Previous approaches to DNA extraction from paraffinembedded tissue have employed either scalpel microdissection of tissue or laser capture microdissection (LCM) for very small specimens. ${ }^{1-6}$ Both techniques have the advantage of being able to separate tumor from normal tissue. Scalpel microdissection employs a stained tissue section to identify tumor or normal regions to similar areas of an unstained section that are carefully scraped from the slide with a scalpel. In LCM, individual clusters of normal or tumor cells can be identified under the microscope, outlined, and collected. Both methods may require cells from up to 20 sections to be dissected in order to obtain adequate DNA for polymerase chain reaction (PCR), and as a result may require the use of a large portion of the specimen. Microdissection and LCM are time consuming and technically challenging. LCM requires special equipment.

Tissue microarrays are a powerful strategy for analysis of gene expression and have become an important research tool. ${ }^{7,8}$ Tissue microarrays contain multiple small cores of tissue harvested from standard paraffin blocks and arrayed into a new paraffin block..$^{9,10}$ This allows hundreds of patients to be analyzed simultaneously from a single section cut from the tissue array. When DNA analysis is simultaneously performed, it is advantageous to use tissue from a site immediately adjacent to those placed into the array.

To construct tissue microarrays, a hematoxylin-eosin (H\&E)-stained slide is prepared from each tissue block, examined under the microscope, and used as a guide to identify the regions of the block that contain either normal cells or cancer cells. These areas are circled on the block with a marking pen, and small cores of tissue are harvested from the regions of interest and used to construct tissue microarrays. Typically, 3 cores of normal tissue and 3 cores of tumor are placed in the new tissue microarray. It is a simple matter to collect additional cores of normal and tumor tissue for DNA extraction at the time the tissue array is built. DNA extraction from such tissue cores has proven to be quick, technically easy, tissue conserving, and adequately enriching for normal and tumor to carry out loss of heterozygosity $(\mathrm{LOH})$ studies. The advantages of the tissue core method for DNA extraction include preservation of most of the original tissue block, the ability to select a specific region of the tissue based on a guide slide (eg, tumor vs normal), the simultaneous creation of a tissue microarray, and the ability to take cores for
DNA extraction adjacent to cores placed in a tissue microarray.

\section{MATERIALS AND METHODS}

A $5-\mu \mathrm{m}$ section was cut from the original tissue block for each specimen prior to harvesting tissue cores. H\&E stained sections were examined microscopically, and areas of tumor and normal tissue were marked. This created a "guide slide" that was compared with the tissue block and used to locate normal and tumor areas on the block from which cores were harvested.

Cores of tissue were collected from paraffinembedded blocks of laryngeal squamous cell carcinoma, normal mucosa and connective tissue, and lymph nodes using a manual tissue puncher/array device (Beecher Instruments, Silver Spring, MD), as previously described. ${ }^{11}$ The instrument consists of a thin-walled stainless steel needle with an inner diameter of $0.6 \mathrm{~mm}$ and a stylet used to transfer the core to its new location. Three cores from both the tumor area and normal area were used from each block to create the tissue array. Approximately 3 to 5 additional cores were harvested from the normal and tumor areas for DNA extraction.

The tissue content of 7 cores collected from different blocks was examined in the following manner. The core was turned on its side and embedded in the new paraffin block. Five-micrometer sections were cut from this new block so that the new sections contained a cross sectional (rectangular) view along the long axis of the core. The H\&Estained sections were examined microscopically to compare the tissue content in the original H\&Estained section with that along the entire length of the core. The percentage of tumor cells in each core was calculated by determining the area of the core containing tumor cells in comparison with the total area of tissue contained within the core.

For DNA extraction, each core was placed in a sterile petri dish and measured to determine the length of tissue contained in the core. Excess paraffin was removed from the bottom of each core and discarded. Samples of tissue, 1, 2, 3, 4, and $5 \mathrm{~mm}$ in length, were cut from replicate cores with a sterile no. 11 scalpel blade. These different lengths were used to evaluate the relative amount of DNA obtained from various tissue core lengths and to determine the optimal length of a tissue core for DNA extraction. The segments of each core were cut into 4 to 5 pieces per millimeter of 


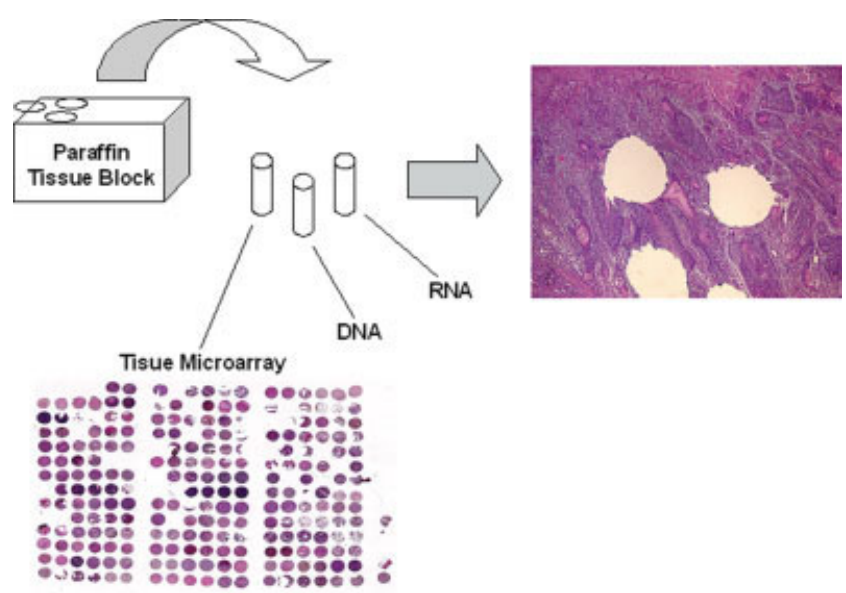

FIGURE 1. Flow diagram for production of tissue arrays and harvesting tissue cores. On the right is a hematoxylin-eosin-stained section from a tissue block from which cores have been punched. [Color figure can be viewed in the online issue, which is available at www.interscience.wiley.com.]

tissue, to expose more surface area for DNA extraction, and transferred to a sterile microcentrifuge tube. DNA was extracted from the cores using the protocol for the Qiagen QIAamp DNA Mini Kit (Qiagen, Valencia, CA) for isolation of genomic DNA from paraffin-embedded tissue. The protocol includes xylene dissolution of paraffin, overnight proteinase $\mathrm{K}$ digestion, and isolation of DNA on a spin column. Prior to the final elution step, elution buffer was warmed to $50^{\circ} \mathrm{C}$, and then $125 \mu \mathrm{L}$ was applied to the column and incubated at $50^{\circ} \mathrm{C}$ for 10 minutes. After centrifuging, the eluate was reapplied to the column and incubated at room temperature for 10 minutes followed by centrifugation. When adequate material was present, the concentration of DNA was determined using OD260 measurements.

The quality and quantity of the DNA was assessed by PCR amplification using 1 and $2 \mu \mathrm{L}$ of template in a reaction volume of $20 \mu \mathrm{L}$ using primers for exon 6 of p53. The PCR reaction mixture consisted of $2 \mu \mathrm{L}$ of $10 \times$ PCR buffer, $1.2 \mu \mathrm{L}$ of $25 \mathrm{mM} \mathrm{MgCl} \mathrm{Mg}_{2}, 0.4 \mu \mathrm{L}$ of $10 \mathrm{~m} M \mathrm{dNTP}, 0.4 \mu \mathrm{L}$ of $10 \mu M$ forward primer, $0.4 \mu \mathrm{L}$ of $10 \mu M$ reverse primer, $0.15 \mu \mathrm{L}$ of Expand High Fidelity enzyme (PE Biosystems, Foster City, CA), 1 or $2 \mu \mathrm{L}$ of template, and water to a total reaction volume of $20 \mu \mathrm{L}$. PCR conditions included incubation at $94^{\circ} \mathrm{C}$ for 7 minutes; 35 cycles of $94^{\circ} \mathrm{C}$ for 50 seconds, $60^{\circ} \mathrm{C}$ for 50 seconds (depending on the melting temperature of the primer sets used), and $72^{\circ} \mathrm{C}$ for 60 seconds; with a final extension period at $72^{\circ} \mathrm{C}$ for 7 minutes. Ten microliters of PCR products were examined using a 1.5\% agarose/ethidium bromide gel.
Forty-three laryngeal cancer specimens were evaluated for p53 gene mutations using Affymetrix p53 GeneChip technology and DNA extracted using the tissue core method. Multiplex PCR samples were prepared for the p53 GeneChip according to the manufacturer's protocol (Affymetrix, Santa Clara, CA). Because DNA from tissue samples is often fragmented, separate PCR reactions for segments of the longest p53 exons (exons 4 and 5 ) were carried out as follows: $3 \mu \mathrm{L}$ template DNA solution, $5 \mu \mathrm{L} 4 \times$ PCR reactants (see manufacturer protocol), $0.4 \mu \mathrm{L}$ forward primer, $0.4 \mu \mathrm{L}$ reverse primer, $0.2 \mu \mathrm{L}$ AmpliTaq Gold (PE Biosystems, Foster City, CA), and $11 \mu \mathrm{L}$ water. PCR conditions included incubation at $95^{\circ} \mathrm{C}$ for 10 minutes, 35 cycles of $95^{\circ} \mathrm{C}$ for 30 seconds, $60^{\circ} \mathrm{C}$ for 30 seconds, and $72^{\circ} \mathrm{C}$ for 45 seconds, and a final extension of $72^{\circ} \mathrm{C}$ for 10 minutes. Ten microliters of multiplex PCR products were examined using a $4 \%$ agarose/ethidium bromide gel. Products of the separate exon reactions were resolved on a $1.5 \%$ agarose/ethidium bromide gel and then purified from the gel using Qiaquick gel extraction kit (Qiagen, Valencia, CA), according to the manufacturer's protocol. For laryngeal carcinoma samples, these products were added to the products of the multiplex PCR prior to proceeding with the remainder of the p53 GeneChip protocol.

LOH at $18 q$ was compared using PCR products from DNA samples of normal and tumor tissue obtained by both microdissection and from tissue cores for 8 different subjects. PCR was run using fluorescent-dye-labeled primers for 8 polymorphic markers along the long arm of chromosome 18q. ${ }^{12}$ Labeled PCR products were transferred to a 96- 

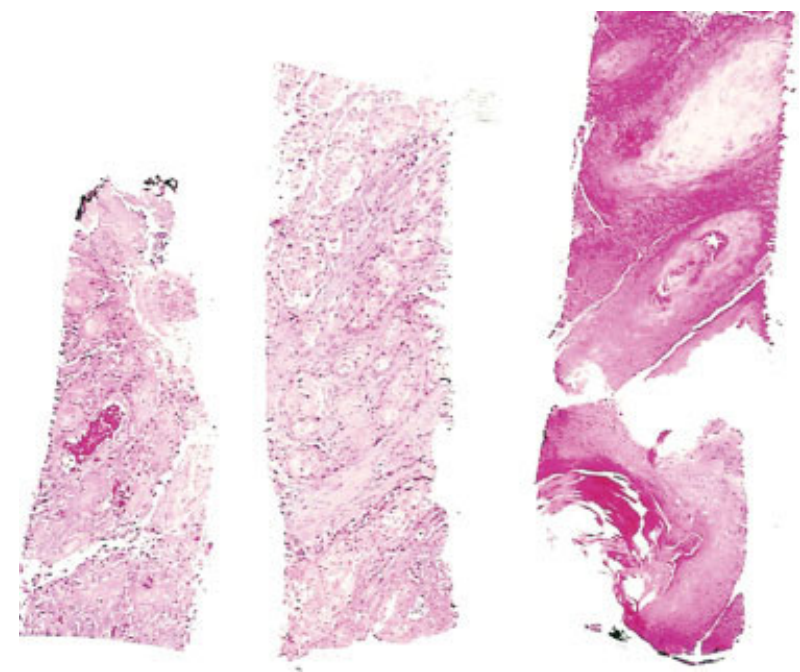

FIGURE 2. Hematoxylin-eosin-stained section of a tissue core cut longitudinally. Section shows tumor cells distributed throughout the core. [Color figure can be viewed in the online issue, which is available at www.interscience.wiley.com.]

well plate. The samples were loaded into the ABI model 7700 Genetic Analyzer and run on a capillary microarray capable of 1 base-pair (bp) resolution. Extraneous peaks resulting from primer dimers and misfires from nonspecific binding were excluded based on the expected size of the amplification products. Chromatograms of the results were printed and assessed for $\mathrm{LOH}$.

\section{RESULTS}

A schematic representation of the sequence followed for collecting tissue cores for microarray construction and DNA extraction is presented in Figure 1. An H\&E-stained section from a block from which cores have been removed for array construction and for DNA extraction is shown in Figure 1. Preservation of the majority of the tissue for future use is evident.

Tissue cores were assessed to determine if the tissue type identified on the "guide slide" is representative of the tissue type through the entire core. Longitudinal sections of cores from 7 different blocks were mounted in new paraffin blocks, sectioned, mounted, stained with H\&E, and analyzed microscopically. In each specimen, the tissue content throughout the core was found to be fairly consistent and to be representative of the tissue type identified on the "guide slide." Microscopic analysis revealed that the tumor cores selected from regions on the guide slide to contain a distri- bution of $\geq 70 \%$ tumor to $\leq 30 \%$ normal cells, equivalent to that typically achievable when using traditional scalpel microdissection from multiple tissue sections. This finding was consistent in all cores analyzed. Representative longitudinal sections from 3 tissue cores are shown in Figure 2. In these cores, the estimated proportion of tumor cells to stromal cells is $70 \%$ to $80 \%$, which is adequate for most types of tumor DNA analyses.

The quantity of DNA isolated from different lengths of the tissue cores was assessed. Squamous cell cancer of the larynx, prostate, and lymph node samples were included in the analysis. Despite the relatively large fragments and reduced surface area of core sections when compared with microdissected tissue, with overnight digestion in proteinase $\mathrm{K}$ of the tissue cores, there was little undigested residue and excellent DNA yields were obtained in proportion to the amount of tissue digested (Figure 3). Early experience with shorter periods of proteinase $\mathrm{K}$ digestion resulted in larger amounts of undigested residue being observed. For prostate and larynx samples, the amount of DNA extracted was stable or appeared to decrease when increasing the tissue core length from 4 to $5 \mathrm{~mm}$. This result may result from less efficient proteinase $K$ digestion with the longer tissue cores. As expected, the best DNA yields were obtained from lymph node samples, which represent the densest distribution of nuclei to volume of the 3 tissue types analyzed.

The quality of the DNA extracted from the cores was evaluated by PCR amplification of a

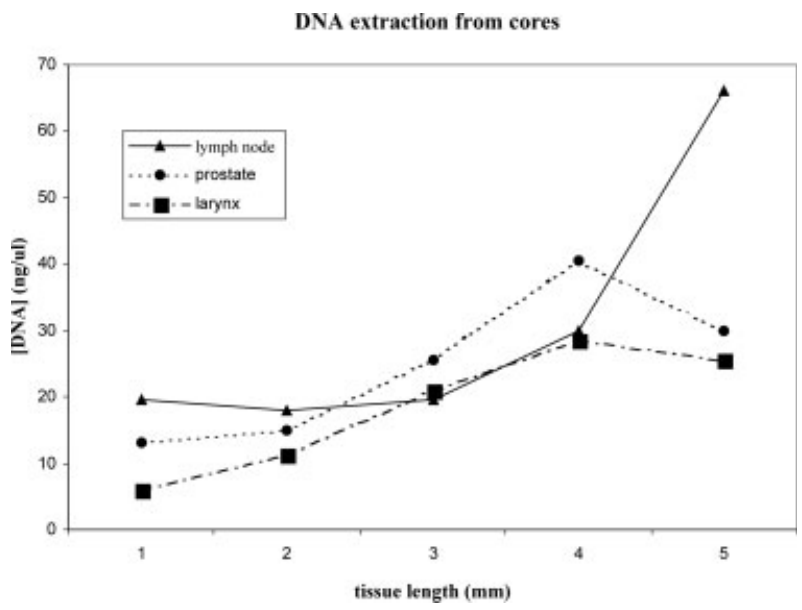

FIGURE 3. Comparison of quantity of DNA obtained versus tissue core length used for DNA extraction. 


\section{PCR result using 1ul (top) or 2 ul (bottom) of template}

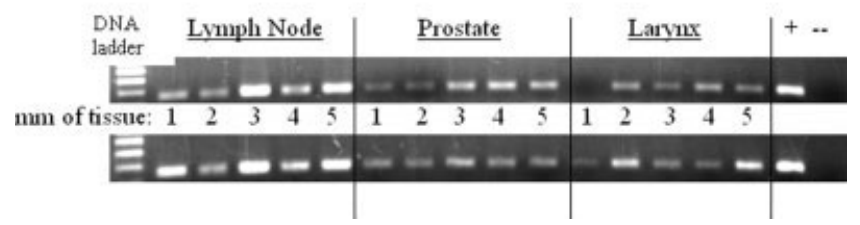

FIGURE 4. Example of results of polymerase chain reaction for exon 6 of p53 utilizing $1 \mu \mathrm{L}$ (top) and $2 \mu \mathrm{L}$ (bottom) of DNA extracted from sections of tissue cores of different lengths.

150-bp segment enclosing exon 6 of the p53 gene (Figure 4). Using $1 \mu \mathrm{L}$ of the DNA solutions, PCR products were visible in every lane for the samples extracted from a $1 \mathrm{~mm}$ core length except for the laryngeal squamous cell carcinoma. When the volume of DNA solution used for PCR was increased to $2 \mu \mathrm{L}$ all lanes contained a visible product.

The DNA isolated by the tissue core method was also tested for use with the p53 GeneChip oligonucleotide microarray (Affymetrix, Santa Clara, CA). The first step in this process is a multiplex PCR, which simultaneously amplifies exons 2 to 11 of p53. As demonstrated in a representative experiment in Figure 5, exons greater than approximately $250 \mathrm{bp}$ in length were not amplified in the laryngeal carcinoma and prostate samples. We postulated that the DNA of the specimens might be more fragmented from the practice of fixation in formalin. The age of the samples, particularly the laryngeal samples, which were over 15 years old, may also have resulted in greater fragmentation of the DNA. A separate PCR reaction using only primers for exon 4 of p53 provided evidence that exon 4 could be amplified in the lymph node, but not in the laryngeal carcinoma or prostate samples. This supported our hypothesis that the DNA from the older formalin-fixed samples had been fragmented to a degree that segments longer than $250 \mathrm{bp}$ (eg, exons 4 and 5) did not produce visible PCR products, although DNA segments from the smaller p53 exons could be amplified. By dividing exon 4 into shorter segments, such as the 146-bp segment illustrated in Figure 5 , the DNA sequence could be amplified without difficulty. Figure 5 shows a PCR product for all samples, including the laryngeal tumor sample, indicating that the DNA for exon 4 is present in the sample, but not as 1 complete segment. To overcome the problem of DNA fragmentation in the older samples and still utilize the p53 GeneChip, exon 4 was amplified as 3 overlapping segments, and exon 5 as 2 overlapping segments. We amplified these smaller segments in separate PCR reactions, purified the products, and added the products to the product of the multiplex PCR reaction prior to proceeding with the p53 GeneChip protocol. This process ensured that exons 2 to 11 were represented in each sample applied to the p53 GeneChip. Forty-three samples were evaluated for p53 mutations using this strategy (data not shown).

An additional assessment of the quality of DNA obtained by the tissue core method was made by comparing samples of normal and tumor DNA isolated from tissue cores with tumor DNA isolated by microdissection for allelic heterozygosity on chromosome arm 18q in 16 individuals. There were no differences in the results obtained using DNA obtained by either technique. In 3 instances in which $\mathrm{LOH}$ was detected using the tissue core method, $\mathrm{LOH}$ was detected using microdissection as well. Similarly, in all cases in which there was no LOH using DNA extracted from the tissue cores, the same result was obtained with microdissected DNA (data not shown).

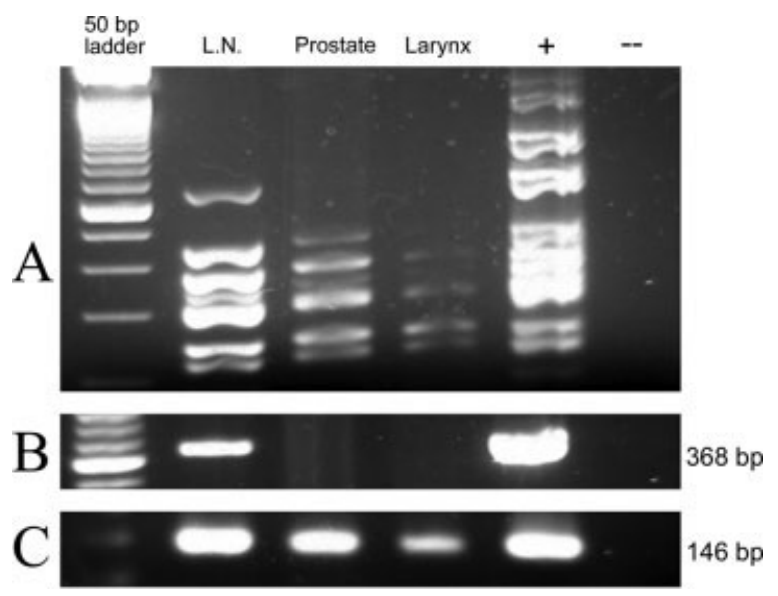

FIGURE 5. Multiplex polymerase chain reaction for exon 4 and 4.1. The results demonstrate amplification of full length exon 4 (row B) in the lymph node sample, but not the larynx or prostate samples. Exon 4.1 (a 176-bp region of exon 4) (row C) is amplified in all samples. 


\section{DISCUSSION}

One of the major advantages of the tissue core method for isolating DNA is the preservation of a large portion of the original tissue sample. As little as $1 \mathrm{~mm}$ of length of a tissue core can provide sufficient DNA for PCR analysis. A single core from blocks that have not been cut numerous times provided sufficient DNA for multiple assays including GeneChip analysis which requires $60 \mu \mathrm{L}$ of PCR reaction product. We were able to successfully use this new method of DNA extraction on specimens from a completed clinical trial in which pretreatment biopsies had been studied numerous times in the past, so the tissue thickness had already been reduced.

Taking cores from paraffin-embedded tissue samples leaves most of the original specimen intact. Sections cut from a tissue block from which tissue cores have been harvested can still be evaluated, as the majority of the original tissue architecture is maintained. The length of the tissue cores obtained from each block is directly related to the thickness of the specimen embedded in paraffin. Longer cores can be obtained from larger specimens and shorter cores from thinner or smaller specimens. For large samples, the effect of taking cores can be almost negligible. For smaller samples, the tissue core technique offers the best method for conserving tissue and preserving the original tissue block for other studies. In comparison, scalpel microdissection of as few as ten $5-\mu \mathrm{m}$ sections from a specimen $5 \mathrm{~mm}$ in diameter would use more tissue than a section $3.5 \mathrm{~mm}$ in length taken from a single tissue core. Analysis has shown that adequate DNA for PCR analysis can be obtained from core segments as small as $1 \mathrm{~mm}$ in length. Additionally, the frustrating problem of air currents or static causing scraped material from conventional section to be repelled from the tube, fall to the floor, or cling to the exterior of the tubes is overcome by the use of the core approach.

The combination of DNA extraction from tissue cores with simultaneous construction of tissue microarrays offers additional advantages. The tissue core for DNA extraction can be taken directly adjacent to a core of tissue that is to be inserted into the tissue microarray. This provides a high degree of correlation between immunohistochemistry and DNA applications. While the ability to separate normal and tumor is possible with other methods of DNA extraction, the tissue core method has the advantage of being simple and quick. This allows for the rapid acquisition of DNA for a variety of applications. The combination of tissue core DNA extraction together with GeneChip technology allows for a rapid turnaround time for both sequence and immunohistochemistry data. In about the same time it takes to construct and stain a tissue microarray, results are available on mutation status using GeneChip technology. DNA extracted by the tissue core method was successfully used to study the mutation status for p53 in 43 laryngeal carcinoma samples.

An important concern regarding the tissue core method of DNA extraction was whether the efficiency of DNA extraction would be reduced due to the larger fragment size. The larger tissue fragments obtained from tissue cores have a lower surface area exposed for proteinase $\mathrm{K}$ digestion than the tissue fragments harvested by microdissection or LCM. This potential problem is easily overcome by using overnight proteinase $\mathrm{K}$ digestion. With this technique, the tissue core method for DNA extraction produces excellent DNA yields with no compromise of DNA quality.

Normal tissue contamination is an important factor when comparing tumor and normal specimens for allelic imbalance and heterozygosity. DNA obtained from carefully mapped tissue cores selected from areas of normal and tumor allows for this type of analysis. The degree of normal tissue contamination of tumor specimens has proven to be equivalent to that obtained by traditional microdissection. In cases in which the specimen contains a high degree of normal tissue contamination and purity is essential, for example, when there is a very marked lymphoid response, LCM may be superior to the tissue core or microdissection techniques. Analysis of $\mathrm{LOH}$ at $18 q$ for 8 different polymorphic markers has shown identical results with DNA obtained from microdissected specimens and from tissue cores. This confirms that the quality of DNA and the degree of normal tissue contamination of the tumor samples extracted by the tissue core technique allows for this type of analysis.

As with any method for extracting DNA from paraffin-embedded tissue, the tissue core method offers the possibility of using tissues that have been stored for many years. The larynx specimens used in this study were originally fixed 15 or more years ago. The ability to successfully extract DNA from preserved tissue samples provides great potential to take advantage of archived tissue sample. Difficulties can arise with older tissue samples when trying to amplify DNA fragments of greater than approximately $250 \mathrm{bp}$. These problems are not the 
result of the method of DNA extraction, but are related to the age of the tissue and the method of fixation. Strategies can be devised to overcome the problem of DNA fragmentation, as we demonstrated with exon 4 and 5 of the p53 gene in these experiments.

Recently, tissue microarray technology has been used to create frozen tissue microarrays. ${ }^{13}$ The primary advantage of such arrays is the preservation of RNA, which is degraded during the fixation required for paraffin embedding. Tremendous potential exists for combining cDNA microarray expression screening analyses with analysis of both DNA and protein on these frozen tissue microarrays. This DNA extraction method can easily be adapted to be used in conjunction with a frozen tissue microarray, allowing correlation of DNA, RNA, and protein data gathered from almost identical sites.

The results of this study demonstrate that the use of DNA extracted from tissue cores for genetic studies is a practical and technically simple companion to tissue microarray profiling. This method has been successfully used to provide rapid and direct correlation of p53 mutation status with expression studies, and in a study looking for allelic imbalance and heterozygosity in head and neck cancer.

Acknowledgments. The authors acknowledge the Prostate Cancer Tissue Microarray Laboratory of the University of Michigan, and the University of Michigan DNA Sequencing Facility.

\section{REFERENCES}

1. Noguchi S, Motomura K, Inaji H, Imaoka S, Koyama H. Clonal analysis of predominantly intraductal carcinoma and precancerous lesions by means of polymerase chain reaction. Cancer Res 1994;54:1849-1853.

2. Emmert-Buck MR, Roth MJ, Zhuang Z, et al. Increased gelatinase A (MMP-2) and cathespin B activity in invasive tumor regions of human colon cancer samples. Am J Pathol 1994;145:1285-1290.

3. Zhuang Z, Bertheau P, Emmert-Buck MR, et al. A microdissection technique for archival DNA analysis if specific cell populations in lesions $<1 \mathrm{~mm}$ in size. Am J Pathol 1995; 146:620-625.

4. Park TW, Felix JC, Wright TC Jr. X chromosome inactivation and microsatellite instability in early and advanced bilateral ovarian carcinomas. Cancer Res 1995;55:4793-4796.

5. Emmert-Buck MR, Bonner RF, Smith PD, et al. Laser capture microdissection. Science 1996;274:998-1001.

6. Rubin MA. Tech. Sight. Understanding disease cell by cell. Science 2002;296:1329,1330.

7. Dhanasekaran SM, Barrette TR, Ghosh D, et al. Delineation of prognostic biomarkers in prostate cancer. Nature 2001;412:822-826.

8. Rubin MA, Zhou M, Dhanasekaran SM, et al. $\alpha$-Methylacyl coenzyme A racemase as a tissue biomarker for prostate cancer. JAMA 2002;287:1662-1670.

9. Kononen J, Bubendorf L, Kallioniemi A, et al. Tissue microarrays for high-throughput molecular profiling of tumor specimens. Nat Med 1998;4:844-847.

10. Hoos A, Cordon-Cardo C. Tissue microarray profiling of cancer specimens and cell lines: opportunities and limitations. Lab Invest 2001;81:1331-1338.

11. Manley S, Mucci NR, De Marzo AM, Rubin MA. Relational database structure to manage high-density tissue microarray data and images for pathology studies focusing on clinical outcome: the prostate specialized program of research excellence model. Am J Pathol 2001;159: 837843.

12. Takebayashi S, Ogawa T, Jung KY, et al. Identification of new minimally lost regions of $18 \mathrm{q}$ in head and neck squamous cell carcinoma. Cancer Res 2000;60:3397-3403.

13. Fejzo MS, Slamon DJ. Frozen tissue microarray technology for analysis of tumor RNA, DNA and proteins. Am J Pathol 2001;159:1645-1650. 